\title{
EFFECT OF BRANCHED-CHAIN AMINO ACIDS, VALINE, ISOLEUCINE AND LEUCINE ON THE BIOSYTHESIS OF BITESPIRAMYCIN 4"-O-ACYLSPIRAMYCINS
}

\author{
Zhen-Lin Li ${ }^{1}$, Yong-Hong Wang*, Ju Chu*, Ying-Ping Zhuang Si-Liang Zhang
}

State Key Laboratory of Bioreactor Engineering, East China University of Science and Technology, Shanghai 200237, China

Submitted: April 10, 2008; Returned to authors for corrections: August 27, 2008; Approved: July 14, 2009.

\begin{abstract}
Bitespiramycin, a group of 4"-O-acylated spiramycins with $4 "$ - $O$-isovalerylspiramycins as the major components, was produced by recombinant spiramycin-producing strain Streptomyces spiramyceticus harboring a 4"-O-acyltransferase gene. The experiment was initially performed in synthetic medium with $0.5 \mathrm{~g} \mathrm{l}^{-1}$ Valine, Isoleucine or Leucine feeding at $36 \mathrm{~h}$ cultivation. When valine was fed, the biological titer of bitespiramycin was $45.3 \%$ higher than that of the control group, but the relative content of total isovalerylspiramycin components decreased by $22.5 \%$. In the case of ilecine, the biological titer of bitespiramycin and the total isovalerylspiramycins alone were $85 \%$ and $72.1 \%$ of the control group, respectively. In contrast, the relative content of other acylated spiramycins increased by $54.41 \%$. However, leucine feeding increased the relative content of total isovalerylspiramycins by $41.9 \%$ while the biological titer of bitespiramycin was nearly equal to that of the control group. The improvement effect of leucine on the biosynthesis of isovalerylspiramycins was further confirmed by feeding of $2.0 \mathrm{~g} \mathrm{l}^{-1}$ leucine to the culture with complex medium. After batch feeding with a total amount of $2.0 \mathrm{~g} \mathrm{l}^{-1}$ leucine to the culture from $70 \mathrm{~h}$ to $90 \mathrm{~h}$, the biological titer of bitespiramycin was almost unreduced, and the final relative content of total isovalerylspiramycins increased from $31.1 \%$ to $46.9 \%$.
\end{abstract}

Key words: bitespiramycin, valine, leucine, isoleucine, biosynthesis

\section{INTRODUCTION}

Bitespiramycin is a group of $4 "-O$-acylated spiramycins with 4 "- $O$-isovalerylspiramycins as the major components (29). Although bitespiramycin has similar antibiotic activity with spiramycin in vitro, the former has superior pharmacokinetic properties compared with spiramycin in vivo. These properties are: quick oral absorption, high tissue permeability, wide distribution in the body. Long in vivo half-life owing to its high lipophilicity. Bitespiramycin is mainly used for the treatment of Gram-positive bacteria infectious diseases, such as upper respiratory tract and urinary system infections $(19,20,21)$.

Bitespiramycin is produced by recombinant Streptomyces spiramyceticus F21 that harbores a 4"-O-acyltransferase gene from S. mycarofaciens 1748 (18). There are a total of 14 components in bitespiramycin owing to the relatively broad substrate specificity of the acyltransferase (10). Thus, during 
the fermentation process of bitespiramycin, the enhancement of the biosynthesis of $4 "-O$-isovalerylspiramycins is a distinctly significant step, as these are the main components responsible for the new pharmacokinetic properties of bitespiramycin.

Amino acids can be used as carbon or nitrogen source in the fermentation of antibiotics. Some amino acids such as valine (Val), isoleucine (Ile) or leucine (Leu) are precursors of some secondary metabolites, and the metabolites derived from them such as acetyl-CoA, propionyl-CoA, isovalerylCoA and butyryl-CoA are precursors of the biosynthesis of macrolide antibiotics (the biosynthesis pathway of bitespiramycin is illustrated in Fig. 1) $(8,14,17,26,28)$.

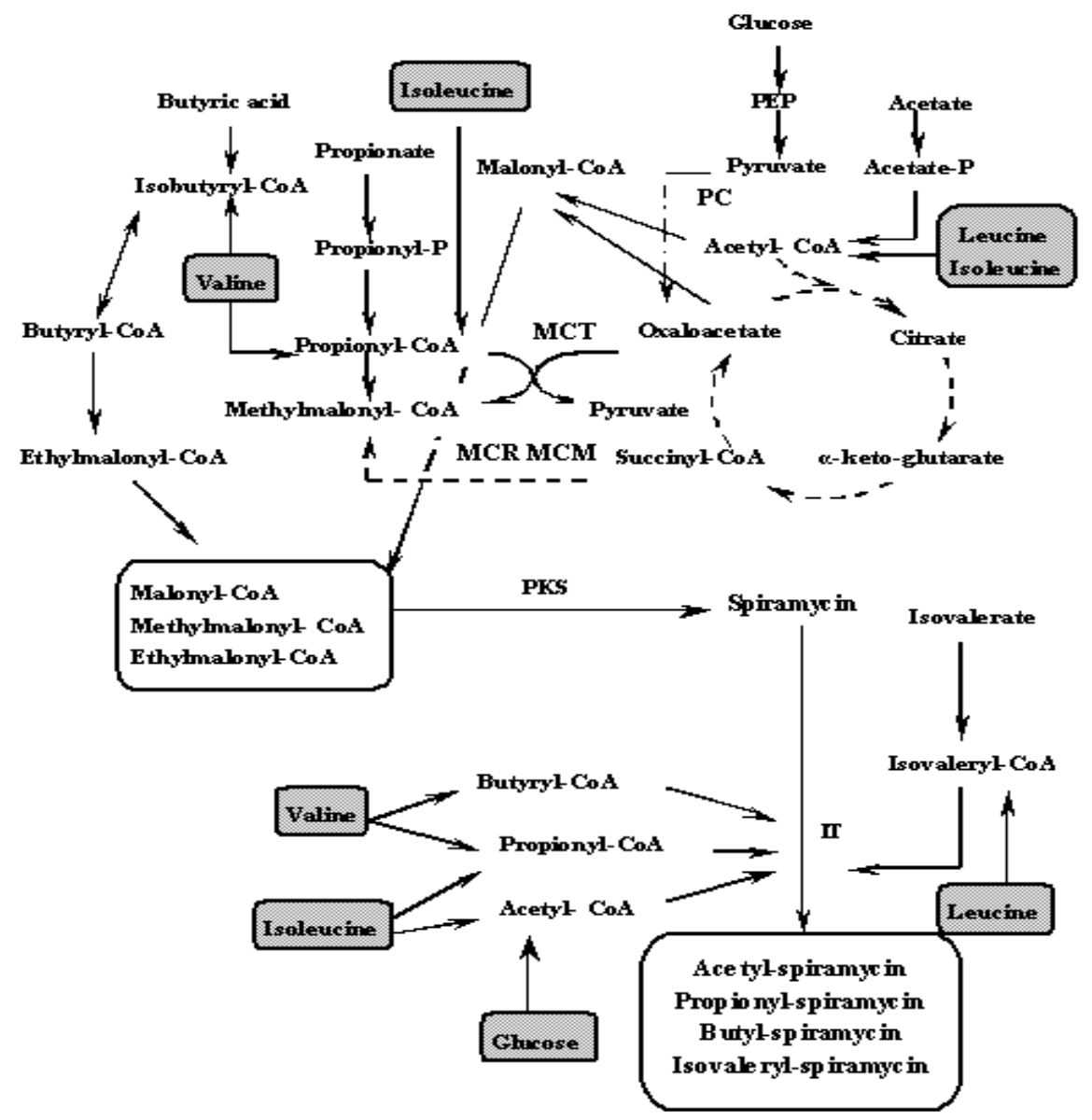

Figure 1. Central metabolic map of S. spiramyceticus.

MCT: Methylmalonyl- CoA Transcarboxylase; MCR: Methylmalonyl- CoA Racemase; MCM: Methylmalonyl-CoA Mutase; PC: Pyruvate Carboxylase; IT: Isovaleryl-CoA Transferase; PKS:Polyketide synthetase; CS: citrate synthase.

This paper focuses mainly on the effect of the supplementation of branched chain amino acids on the composition and biological titer of bitespiramycin. We evaluate whether supplementation with Val, Leu or Ile in the culture with synthetic media have different effects on the composition and biological titer of bitespiramycin, and discuss the mechanism of the three amino acids by analyzing the process data of organic acids and related enzyme activities. A comparison study of single and batch supplementation of Leu into the fermentation process with complex media is also made to verify the results derived from the culture with a defined medium. 


\section{MATERIALS AND METHODS}

\section{Microorganism and cultivation conditions}

In all the experiments, a gene-engineered strain WSJ-1195 constructed by integrating isovaleryltransferase gene from a carbomycin-producing strain S. mycarofaciens 1748 into a spiramycin-producing strain Streptomyces spiramyceticus F21 was used (18).

The primary seed culture and secondary seed culture used the same seed medium containing $\left(\mathrm{g} \mathrm{l}^{-1}\right)$ glucose $1 \mathrm{~g}$, corn starch $3 \mathrm{~g}$, soybean meal $2 \mathrm{~g}, \mathrm{NaCl} 0.4 \mathrm{~g}$ and $\mathrm{CaCO}_{3} 0.5$ g.

The synthetic medium contained $\left(\mathrm{g} \mathrm{l}^{-1}\right)$ : dextrin 50, $\mathrm{NH}_{4} \mathrm{NO}_{3} 2 \mathrm{KH}_{2} \mathrm{PO}_{4} 0.65 \mathrm{CoCl}_{2} 0.0005 \mathrm{MgSO}_{4} 5.5 \mathrm{NaCl} 10$ and $\mathrm{CaCO}_{3} 5$.

The fermentation medium contained $\left(\mathrm{g} \mathrm{l}^{-1}\right)$ : glucose 50, fish meal 23, starch 60, $\mathrm{NH}_{4} \mathrm{NO}_{3} 6 \square \mathrm{KH}_{2} \mathrm{PO}_{4} 0.65 \mathrm{MgSO}_{4} 5.5$ $\mathrm{NaCl} 10$ and $\mathrm{CaCO}_{3} 5$.

The spore solution of recombinant Streptomyces spiramyceticus WSJ-1-195 in 20\% glycerol was stored in -80 and the count was more than $5 \times 10^{6}$ spore $\mathrm{ml}^{-1}$. For seed cultivation, one $\mathrm{ml}$ spore solution was inoculated into a primary 250-ml shake flask containing $50 \mathrm{ml}$ seed medium. After $48 \mathrm{~h}$ of incubation at $28,10 \mathrm{ml}$ of primary seed culture was inoculated into a secondary 500-ml shake flask containing $100 \mathrm{ml}$ seed medium. After $24 \mathrm{~h}$ of incubation at $28,4 \mathrm{ml}$ of secondary seed culture was inoculated into a 500$\mathrm{ml}$ fermentation flask containing $50 \mathrm{ml}$ fermentation medium, which was shaken at 220 revolutions per minute and 28 for $96 \mathrm{~h}$ of fermentation.

For the feeding method of the three amino acids, $0.5 \mathrm{~g} \mathrm{l}^{-1}$ Val, Leu or Ile was treated with ultraviolet radiation for $12 \mathrm{~h}$, and then the sterilized Val, Leu or Ile was supplemented into different fermentation flasks to obtain the final defined concentration of each of the amino acids.

Feeding method of the Leu in the culture with complex medium: (1) $2.0 \mathrm{~g} \mathrm{l}^{-1}$ Leu was supplemented at one time at 72 $\mathrm{h}$ of fermentation; (2) at $72 \mathrm{~h}$ of fermentation, a total of $2.0 \mathrm{~g}$ $\mathrm{l}^{-1}$ Leu was fed at an interval of $6 \mathrm{~h}(72 \mathrm{~h}, 80 \mathrm{~h}$ and $90 \mathrm{~h}$ of fermentation).

\section{Total sugar, ammonium ion, dry cell weight and the biological titer determination}

Total sugar concentrations in the medium were determined using dinitrosalicylic acid (DNS) method (15). The supernatant of the fermentation broth obtained after centrifugation was boiled with $6 \mathrm{~N} \mathrm{HCl}$ for $10 \mathrm{~min}$ and neutralized by equal volumes of $6 \mathrm{~N} \mathrm{NaOH}$. The hydrolysate was analyzed using dinitrosalicylic acid (DNS) method. The ammonium ion in the defined medium was determined using a method described by Weatherburn (25), while $\mathrm{NH}_{2}-\mathrm{N}$ in the complex medium was analyzed by Foreman's formaldehyde titration method. Determination of dry cell weight (DCW) was performed using the method described by Lebrihi (12). Biological titer was estimated by the conventional disc method using Bacillus pumilus as test microorganism and acetylspiramycin as the standard (1).

\section{Components and organic acids measurements by HPLC}

The fermentation broth of bitespiramycin was centrifuged at 3000 revolutions per minute for $10 \mathrm{~min}$ and the supernatant was stored at -20 for further use. The supernatant was centrifuged again at 12,000 revolutions per minite for 3 min and passed through the filtration membrane $(0.45 \mu \mathrm{m})$ for the measurement of bitespiramycin components, acetic acid, propionic acid, butyric acid and isovaleric acid by highperformance liquid chromatography (HPLC) system (HP1100, Agilent Tech, USA).

The determination of bitespiramycin components was conducted using a $\mathrm{C}_{18}$ column $(150 \mathrm{~mm} \times 4.6 \mathrm{~mm}, 5 \mu \mathrm{m})$ at 25 . The mobile phase was the mixture solution of $0.083 \mathrm{~mol}$ $\mathrm{L}^{-1}$ phosphoric acid water solution and methanol (53:47) at a flow rate of $1 \mathrm{ml} \mathrm{min}^{-1}$ and detected at $234 \mathrm{~nm}$. The standard of bitespiramycin was provided by the Institute of Medicinal Biotechnology (IMB) of the Chinese Academy of Medical Sciences. 
The determination of acetic acid and propionic acid in the broth was conducted using a $\mathrm{C}_{8}$ column $(250 \times 4.6 \mathrm{~mm}, 5 \mu \mathrm{m})$ at 30 . The mobile phase was the the mixture solution of 0.01 mol $\mathrm{l}^{-1}$ phosphoric acid water solution (adjusted to $\mathrm{pH}$ 2.3 \pm 0.1 with $\mathrm{NaH}_{2} \mathrm{PO}_{4}$ ) and methanol (98.5:1.5) at the flow rate of $1 \mathrm{ml} \mathrm{min}{ }^{-1}$, and detected at $210 \mathrm{~nm}$.

The determination of butyric acid and isovaleric acid in the broth was conducted using a $\mathrm{C}_{18}$ column $(150 \times 4.6 \mathrm{~mm}, 5$ $\mu \mathrm{m})$ at 30 . The mobile phase was the the mixture solution of $0.01 \mathrm{~mol} \mathrm{l}^{-1}$ phosphoric acid water solution (adjusted to $\mathrm{pH}$ $2.3 \pm 0.1$ with $\left.\mathrm{NaH}_{2} \mathrm{PO}_{4}\right)$ and methanol (60:40) at the flow rate of $1 \mathrm{ml} \mathrm{min}$, and detected at $210 \mathrm{~nm}$. The standard of acetic acid, propionic acid, butyric acid and isovaleric acid was purchased from Sigma.

\section{Determination of intracellular pyruvate acid by HPLC}

The mycelia were centrifuged at 4 and $4000 \mathrm{r} \mathrm{min}^{-1}$ for 5 min, washed with $0.88 \%\left(\mathrm{~m} \mathrm{v}^{-1}\right)$ normal saline twice, and then resuspended in $5 \mathrm{ml}$ of $0.01 \mathrm{~mol} \mathrm{l}^{-1}$ phosphoric acid water solution. The mixture solution was disrupted with French Pressure (Xinzhi, Shanghai) at $200 \mathrm{Mpa}$, after which the lysate was centrifuged at 4 and $12000 \mathrm{r} \mathrm{min}^{-1}$ for $30 \mathrm{~min}$. The supernatant was used for determining intracellular pyruvate acid. The measurement conditions for HPLC were the same as those for the extracellular acetic acid and propionic acid described above.

\section{The activities of pyruvate carboxylase (PC) and citrate synthase (CS) measurement}

The mycelia were collected by freeze centrifugation at 4 and $4000 \mathrm{r} \mathrm{min}^{-1}$ for $5 \mathrm{~min}$, washed with $10 \%\left(\mathrm{~m} \mathrm{v}^{-1}\right)$ sucrose solution for two times, and then resuspended in $5 \mathrm{ml}$ of 0.05 mol $1^{-1}$ Tris- $\mathrm{HCl}$ buffer solution ( $\left.\mathrm{pH} 7.4\right)$ containing $20 \%$ glycerol. The mixture solution was disrupted with French Pressure (Xinzhi, Shanghai) at $200 \mathrm{Mpa}$, after which the lysate was centrifuged at 4 and $12000 \mathrm{r} \mathrm{min}^{-1}$ for $30 \mathrm{~min}$. The supernatant was used for the assay of enzymatic activity.

The activities of PC and CS were measured according to the reference papers $(6,13)$. One unit $(U)$ of a specific enzyme was defined as $1 \mu \mathrm{mol}$ of catalysate produced per min. The protein content was determined by Coomassie brilliant blue G250 staining method, and calculated according to the standard curve of bovine serum albumin.

\section{RESULTS}

\section{The effects of Val, Ile or Leu on the yield and the composition of bitespiramycin}

The supplementation of Val, Ile or Leu during the fermentation process of bitespiramycin influenced the growth of microorganism and the biosynthesis of bitespiramycin. After supplementation with amino acids, the biomass was a little lower than that of the control group, the increase in the titer of bitespiramycin was nearly stopped ( $48 \mathrm{~h}$ ), and the inter-group differences increased with the ongoing fermentation process. In the culture supplemented with Val, the titer of bitespiramycin increased significantly after $12 \mathrm{~h}$ adaptation, higher than that of the control group by $45.3 \%$ at the end of fermentation. As for Ile, the titer increased slowly, and towards the end of the fermentation process, was only $85 \%$ of the control group. For Leu, the titer of bitespiramycin was almost equal to that of the control group.

Table 1 shows that after supplementation with Leu, the contents of isovalerylspiramycin II and isovalerylspiramycin III increased to $141.2 \%$ and $150.4 \%$ of the control group, respectively, and the content of total isovalerylspiramycins increased by $42.3 \%$. The content of spiramycins decreased significantly, and the contents of other minor acylated components decreased as well.

After supplementation with Ile, the relative content of propionylspiramycin III, acetylspiramycin III and isobutyrylspiramycin II increased by $126.1 \%, 53 \%$ and $296 \%$, respectively, while the contents of spiramycins and isovalerylspiramycins decreased to only $66.2 \%$ and $72.1 \%$ of that of the control group, respectively.

After supplementation with $\mathrm{Val}$, the relative content of spiramycins and butyrylspiramycin increased, the most 
Zhen-Lin, L. et al.

apparent changes being the increase of the relative content of spiramycin I and spiramycin III. The relative contents of total isovalerylspiramycins and other acylated components decreased by $22.5 \%$ and $27.5 \%$ while the relative contents of total spiramycins increased by $54.5 \%$, respectively.

Table 1. Effects of Val, Ile or Leu on the components of bitespiramycin

\begin{tabular}{lcccc}
\hline \multicolumn{1}{c}{ Component } & \multicolumn{3}{c}{ Content (\%) } \\
\cline { 2 - 5 } & Valine & Isoleucine & Leucine & Control \\
\hline Isovalerylspiramycin & $8.22 \pm 0.21$ & $3.26 \pm 0.22$ & $2.78 \pm 0.12$ & $2.92 \pm 0.11$ \\
Isovalerylspiramycin & $5.61 \pm 0.28$ & $5.90 \pm 0.25$ & $14.12 \pm 0.67$ & $10.00 \pm 0.54$ \\
IsovalerylspiramycinIII & $10.25 \pm 0.86$ & $13.25 \pm 0.71$ & $27.34 \pm 0.92$ & $18.18 \pm 0.84$ \\
Total isovalerylspiramycins & $24.08 \pm 0.45$ & $22.41 \pm 0.45$ & $44.24 \pm 0.64$ & $31.09 \pm 0.75$ \\
Acetylspiramycin III & $8.30 \pm 0.42$ & $18.40 \pm 0.63$ & $8.71 \pm 0.54$ & $12.02 \pm 0.43$ \\
Propionylspiramycin & $2.68 \pm 0.12$ & $3.65 \pm 0.27$ & $3.12 \pm 0.14$ & $4.06 \pm 0.12$ \\
Propionylspiramycin III & $5.57 \pm 0.26$ & $23.67 \pm 0.65$ & $9.87 \pm 0.25$ & $10.47 \pm 0.24$ \\
Butylspiramycin & $6.79 \pm 0.23$ & $8.69 \pm 0.65$ & $2.16 \pm 0.18$ & $2.17 \pm 0.18$ \\
Other acyl-Spiramycin & $23.34 \pm 0.37$ & $54.41 \pm 1.54$ & $23.86 \pm 0.95$ & $28.72 \pm 0.83$ \\
Total spiramycin & $48.92 \pm 0.69$ & $20.97 \pm 0.52$ & $23.95 \pm 0.89$ & $31.67 \pm 0.66$ \\
\hline A total of 0.5 g $1^{-1}$ Val, Ile or Leu was fed at $36 \mathrm{~h}$ of bitespiramycin fermentation. The culture without amino acid \\
feedings was used as control. The content of other acylated spiramycins was calculated by (100-Total spiramycins- \\
Total isovalerylspiramycins).
\end{tabular}

After supplementation with $\mathrm{Val}$, the relative content of spiramycins and butyrylspiramycin increased, the most apparent changes being the increase of the relative content of spiramycin I and spiramycin III. The relative contents of total isovalerylspiramycins and other acylated components decreased by $22.5 \%$ and $27.5 \%$ while the relative contents of total spiramycins increased by $54.5 \%$, respectively.

\section{The effects of Val, Ile or Leu on glycometabolism}

Fig. 2A shows that the $\mathrm{pH}$ of the culture broth did not increase after supplementation with $\mathrm{Val}$, but was 0.1-0.2 $\mathrm{pH}$ higher than that of the control group for Leu and Ile. Fig. 2B shows that the concentrations of ammonium ion in the fermentation broth $12 \mathrm{~h}$ after supplementation with Ile and Leu were $4.0 \mathrm{mmol} \mathrm{l}^{-1}$ and $3.5 \mathrm{mmol} \mathrm{l}^{-1}$, respectively, which eventually decreased slightly. However, after supplementation with $\mathrm{Val}$, the concentration of ammonium ion in the fermentation broth became almost the same as that of the control group, i.e., maintained at about $1.0 \mathrm{mmol}^{-1}$. These results indicate that Streptomyces spiramyceticus utilized and metabolized the three amino acids in different ways.

Fig. 3A shows that after supplementation with Val, the glucose consumption rate increased, the residual total sugar in the fermentation broth decreasing rapidly from $23.4 \mathrm{~g} \mathrm{l}^{-1}$ to $11.4 \mathrm{~g} \mathrm{l}^{-1}$ from $48 \mathrm{~h}$ to $60 \mathrm{~h}$, at this phase the glucose consumption rate reached the highest level of $1.0 \mathrm{~g} \mathrm{l}^{-1} \mathrm{~h}^{-1}$. After supplementation with Ile, the glucose consumption rate was only 0.1-0.2 $\mathrm{g} \mathrm{l}^{-1} \mathrm{~h}^{-1}$, which was apparently lower than that of the control group, whereas after supplementation with Leu, the glucose consumption rate was $0.3-0.5 \mathrm{~g} \mathrm{l}^{-1} \mathrm{~h}^{-1}$, which was similar to that of the control group. It is obvious that the glucose consumption rate is closely correlated to the titer of bitespiramycin. 
It is shown in Fig. 3B that after supplemention with Val, Ile or Leu, the concentration of intracellular pyruvic acid was in the range of 2.7-4.3 $\mathrm{mmol} \mathrm{l}^{-1}, 0.5-1.0 \mathrm{mmol} \mathrm{l}^{-1}$, and 0.9-2.4 mmol $1^{-1}$, respectively, which was higher, lower and roughly
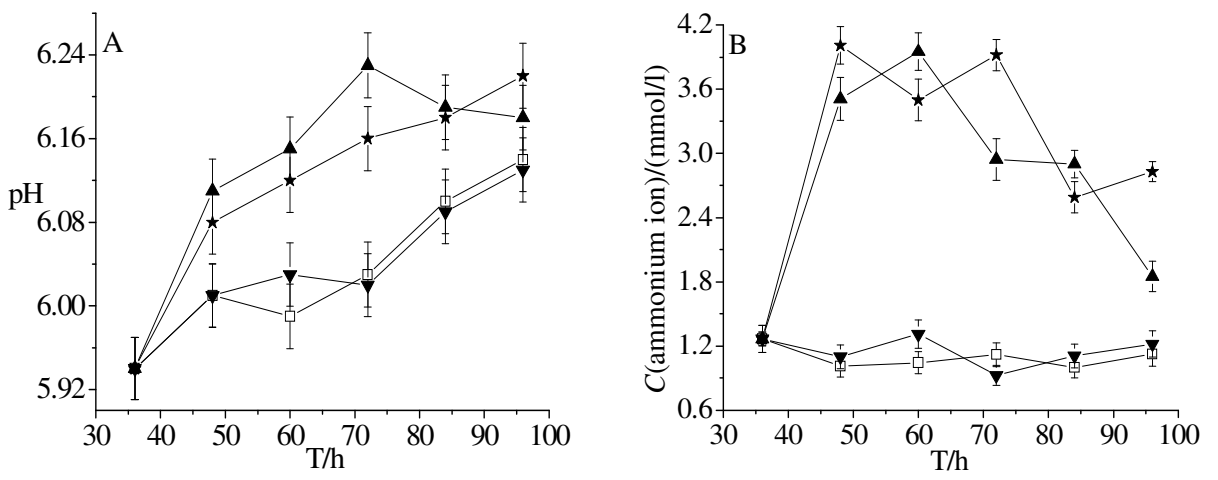

$\square \square-$ control $-\boldsymbol{\nabla}$ - addition of Val- $\mathbf{\Delta}$-addition of Ile $-\square \square$ addition of Leu

Figure 2. Profiles of $\mathrm{pH}$ and ammonium concentration in bitespiramycin fermentation with $0.5 \mathrm{~g} \mathrm{l}^{-1} \mathrm{Val}$, Ile or Leu supplemented at $36 \mathrm{~h}$.
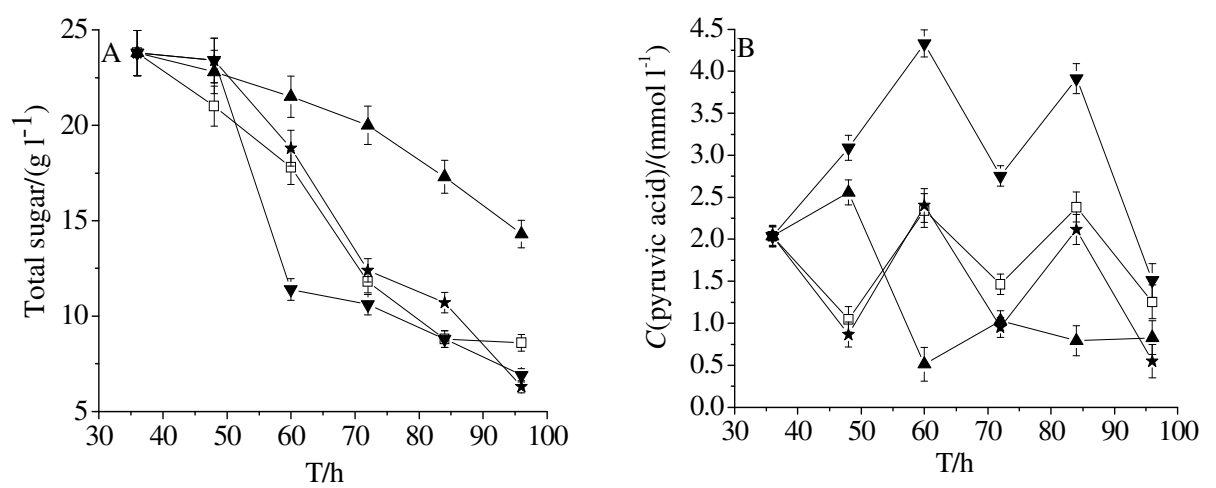

$\square \square-$ control $-\boldsymbol{\nabla}$ - addition of Val- $\mathbf{\Delta}$-addition of Ile $-\square \square$ addition of Leu

Figure 3. Profiles of total sugar and intracellular pyruvic acid concentration in bitespiramycin fermentation with $0.5 \mathrm{~g} \mathrm{l}^{-1} \mathrm{Val}_{\text {, }}$ Ile or Leu supplemented at $36 \mathrm{~h}$.

Fig. 4 shows the change of activities of PC and CS after supplementation with the three amino acids. PC converts pyruvic acid into oxaloacetate, while CS is the key enzyme of TCA cycle, and the activity of CS may reflect the intensity of
TCA cycle (11), which is directly correlated to the primary metabolism of microorganisms. As displayed in Fig. 4A, at $48 \mathrm{~h}$ and $60 \mathrm{~h}$, the activity of PC in the culture with Val increased to $120 \%$ and $195.5 \%$ of that of the control group, 
respectively. After supplementation with Ile, the activity of PC decreased to $62.6 \%$ and $72.5 \%$, respectively. For Leu, the activity of PC did not manifest obvious changes. As shown in Fig. 4B, at $48 \mathrm{~h}$ and $60 \mathrm{~h}$, the activity of CS in the culture

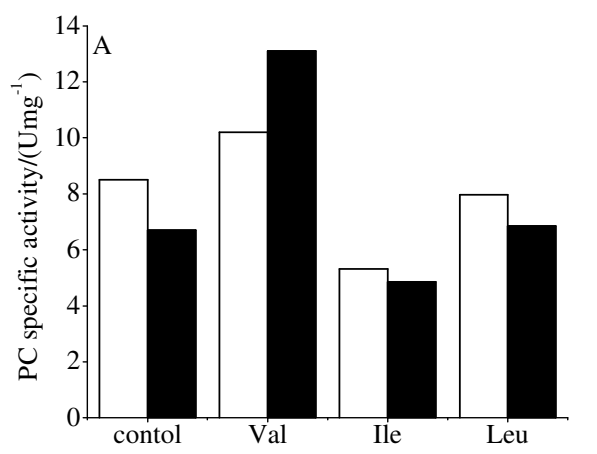

$\square 48 \mathrm{~h}$ with Val decreased to $42 \%$ and $23.2 \%$ of that of the control group, respectively. After supplementation with Ile, the activity of CS decreased to $47.3 \%$ and $51.7 \%$, respectively. For Leu, the activity of CS did not manifest obvious changes.

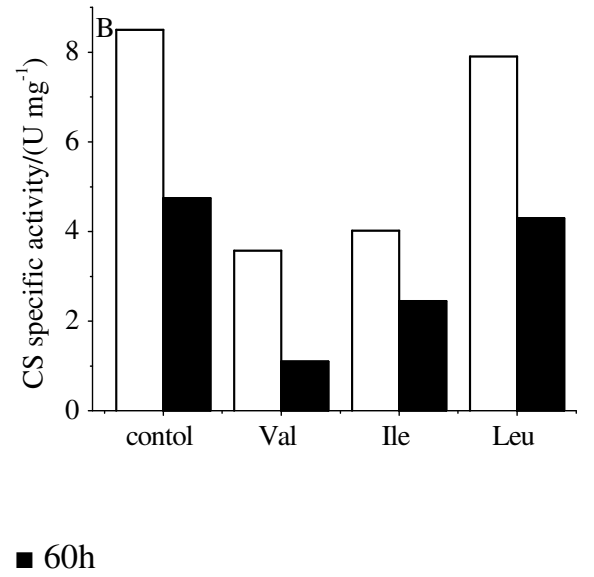

Figure 4. Effect of $0.5 \mathrm{~g} \mathrm{l}^{-1} \mathrm{Val}$, Ile or Leu supplemented at $36 \mathrm{~h}$ on the pyruvate carboxylase (PC) and citrate synthase (CS) activities at $48 \mathrm{~h}$ and $60 \mathrm{~h}$, respectively

The above results indicate that after supplementation with Val, both the activity of PC and concentration of intracellular pyruvic acid increased substantially. Intracellular pyruvic acid is mainly converted into acetyl-CoA and oxaloacetic acid (which is one of the main substrates of the biosynthesis of malonyl-CoA and methylmalonyl-CoA). The increase in the activity of PC and the concentration of intracellular pyruvic acid may have resulted in more sufficient direct precursors pool for the biosynthesis of macro ring, thus the increase in the titer of bitespiramycin.

After supplementation with Ile, both PC and CS activity decreased, and the concentration of intracellular pyruvic acid decreased at the same time, implying that both the primary and secondary metabolism of the biomass became weaker. This resulted in the reduction of biomass and titer of bitespiramycin. However, after supplementation with Leu, activities of both PC and CS were almost the same as those of the control group, resulting in the absence of notable decreases in bitespiramycin titers, but a great increase in the amount of total isovalerylspiramycin components (especially isovalerylspiramycin III) was observed.

\section{The effects of Val, Ile or Leu on the extracellular short-} chain fatty acids

As shown in Fig. 5A, after supplementation with Val and Ile, the concentration of extracellular acetic acid increased rapidly and displayed a constant upward trend. But after supplementation with Leu, the concentration of extracellular acetic acid did not obviously change, and the accumulation of extracellular acetic acid did not occur until $72 \mathrm{~h}$ later.

A comparison of the changes in extracellular propionic acid was shown in Fig. 5B. The concentration of extracellular propionic acid was almost equal to that of the control group after supplementation with Leu while it was higher than that of the control after Val or Ile supplementation.

The change trend of isovaleric acid is depicted in Fig. 5C. After supplementation with Leu, the concentration of extracellular isovaleric acid increased rapidly to the highest 
level at $1.66 \mathrm{~mol} \mathrm{l}^{-1}$ at $84 \mathrm{~h}$, and then decreased to $0.9 \mathrm{mmol} \mathrm{l}^{-}$ ${ }^{1}$. But after supplementation with $\mathrm{Val}$ or Ile, the concentration of extracellular isovaleric acid became slightly lower than that of the control group.

The behavior of extracellular butyric acid is shown in Fig. 5D. After supplementation with Val, the concentration of extracellular butyric acid increased to $14 \mathrm{mmol} \mathrm{l}^{-1}$ at $48 \mathrm{~h}$, then decreased quickly, and reached a plateau within the range of 6.2-8.6 $\mathrm{mmol} \mathrm{l}^{-1}$. After supplementation with Ile, the concentration of extracellular butyric acid became consistently higher than that of the control group, and was maintained within the range of 13-19 $\mathrm{mmol} \mathrm{l}^{-1}$. The change in the trend of the concentration of extracellular butyric acid after supplementation with Leu was similar to that of the control group.
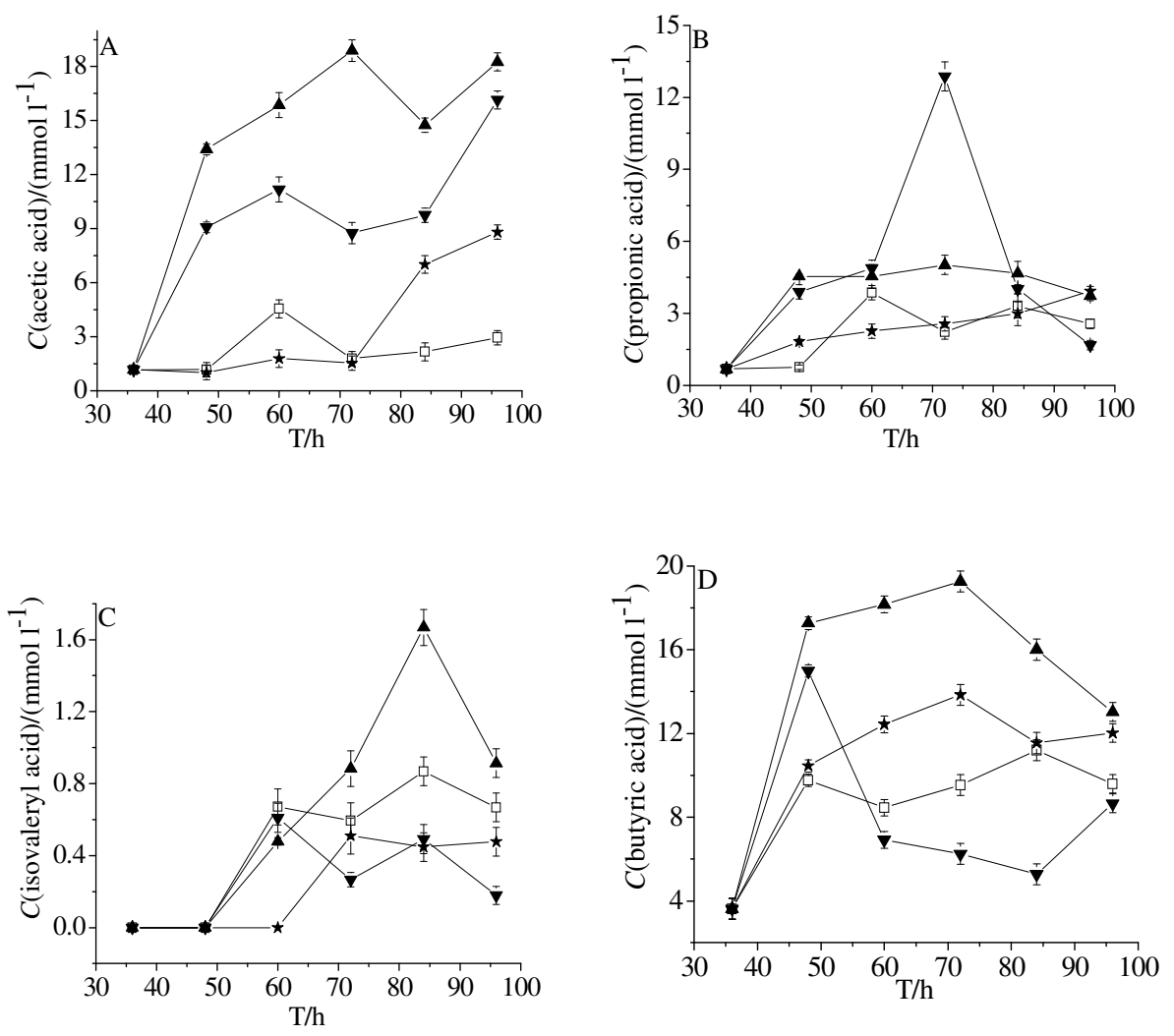

$\square \square-$ control $-\boldsymbol{\nabla}$ - addition of Val- $\mathbf{\Delta}$-addition of Ile $-\square \square$ addition of Leu

Figure 5. Profile of short-chain fatty acid concentrations in bitespiramycin fermentation with $0.5 \mathrm{~g} 1^{-1} \mathrm{Val}$, Ile or Leu supplemented at $36 \mathrm{~h}$

After supplementation with Val, the glucose consumption rate, acetic acid, and propionic acid increased. The increase in the activity of PC showed that more intracellular pyruvic acid can be converted into oxaloacetic acid. Oxaloacetic acid is involved in the biosynthesis of malonyl-CoA and methylmalonyl-CoA, which provide the direct precursors for the biosynthesis of lactone ring, thus improving the biological titer of bitespiramycin.

After supplementation with Leu, butyric acid accumulated slightly, while the change trends in the concentrations of acetic acid and propionic acid were not significantly affected. The biological titer of bitespiramycin 
did not obviously vary, either. The relative content of isovalerylspiramycin components increased significantly, indicating that isovaleryl-CoA produced by the catabolism of Leu can be introduced at the 4 position of spiramycins.

\section{Effecst of Leu feeding on the composition of bitespiramycin in the culture with complex medium}

Fig. 6 shows that both single and multiple batch supplements of Leu obviously improved the composition of bitespiramycin. At $96 \mathrm{~h}$ post inoculation (at the end of fermentation), the content of total isovalerylspiramycins was $46.87 \%$ and $39.42 \%$ by batch or single modes compared to $31.14 \%$ in the culture without Leu. After single supplementation with Leu, the biological titer of bitespiramycin was $74 \%$ compared to that of the control group. But the biological titer of bitespiramycin in the group with batch supplementation with Leu was similar to that of the control group, reaching $91 \%$ of the control at the end of fermentation.

The above result shows that batch supplementation with Leu is superior to single supplementation with Leu for the improvement of the composition of bitespiramycin. Specifically, batch supplementation with Leu compensated for the loss of the biological titer of bitespiramycin that occurred after single supplementation with Leu.
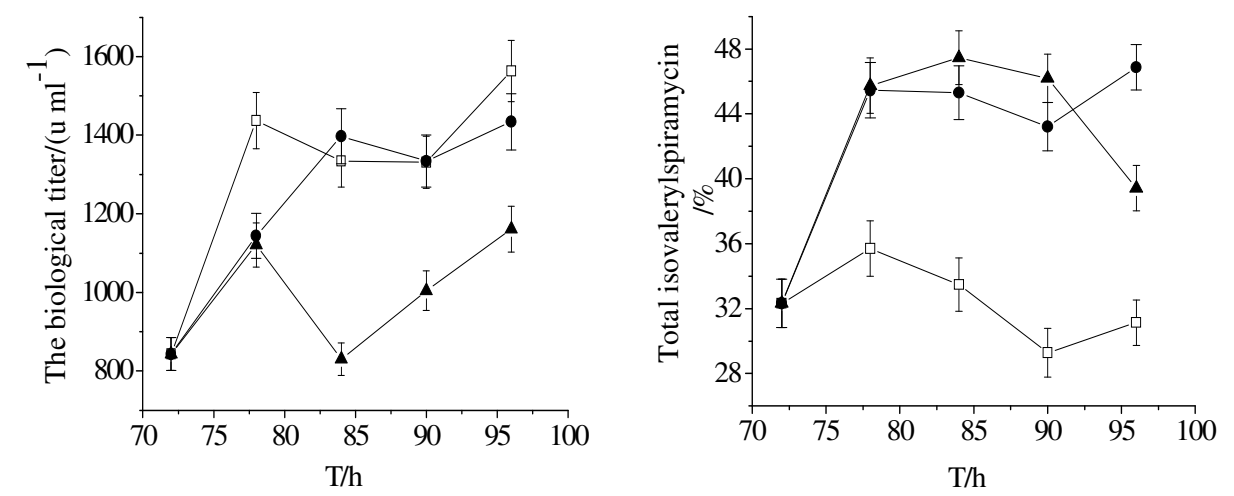

$\square \square-$ control $-\boldsymbol{\Delta}-$ Leu fed by single addition - $\bullet-$ Leu fed by multiple batch addition

Figure 6. Effect of Leu feeding mode on bitespiramycin fermentation with complex medium. Single addition: $2.0 \mathrm{~g} 1^{-1}$ Leu was supplemented at one time at $72 \mathrm{~h}$ of fermentation. Multiple batch addition: at $72 \mathrm{~h}$ of fermentation, a total of $2.0 \mathrm{~g} \mathrm{l}^{-1}$ Leu was fed at an interval of $6 \mathrm{~h}(72 \mathrm{~h}, 80 \mathrm{~h}$ and $90 \mathrm{~h}$ of fermentation).

Fig. 7 shows that single supplementation with $2.0 \mathrm{~g}^{-1}$ Leu at $72 \mathrm{~h}$ of fermentation slowed down the utilization of sugar, with the glucose consumption rate being $1.1 \mathrm{~g} \mathrm{l}^{-1} \mathrm{~h}^{-1}$ from $72 \mathrm{~h}$ to $84 \mathrm{~h}$, lower than that $\left(1.6 \mathrm{~g} \mathrm{l}^{-1} \mathrm{~h}^{-1}\right)$ of the control group. In addition, the $\mathrm{pH}$ level and amino nitrogen increased. But after batch supplementation of Leu, the utilization profile of total sugar became similar to that of the control group. The amino nitrogen did not accumulate, and the $\mathrm{pH}$ became higher than that of the control group but lower than that of the group with single supplementation of Leu. The pyruvic acid concentration after single and multiple batch supplementation of Leu increased from $4.7 \mathrm{mmol}^{-1}$ to $9.8 \mathrm{mmol} \mathrm{l}^{-1}$ and $12.82 \mathrm{mmol}^{-1}$, respectively, but the pyruvic acid concentration of the control group increased to 16.23 mmol $1^{-1}$.

By comparing the metabolism parameter changes after single and batch supplementation with Leu, it has been found that after single supplementation with Leu, the utilization of 
glucose was inhibited to some extent, the amino nitrogen obviously increased, and the $\mathrm{pH}$ had a corresponding sharp increase. After batch supplementation with Leu, the utilization rate of glucose and amino nitrogen did not change greatly. The reason may be that after single supplementation with Leu, large amount of ammonium ion produced by deamination of Leu brought about a notable increase in amino nitrogen, resulting in the sharp change in $\mathrm{pH}$ and the inhibition of the utilization of glucose, thus the decrease in biological titer.
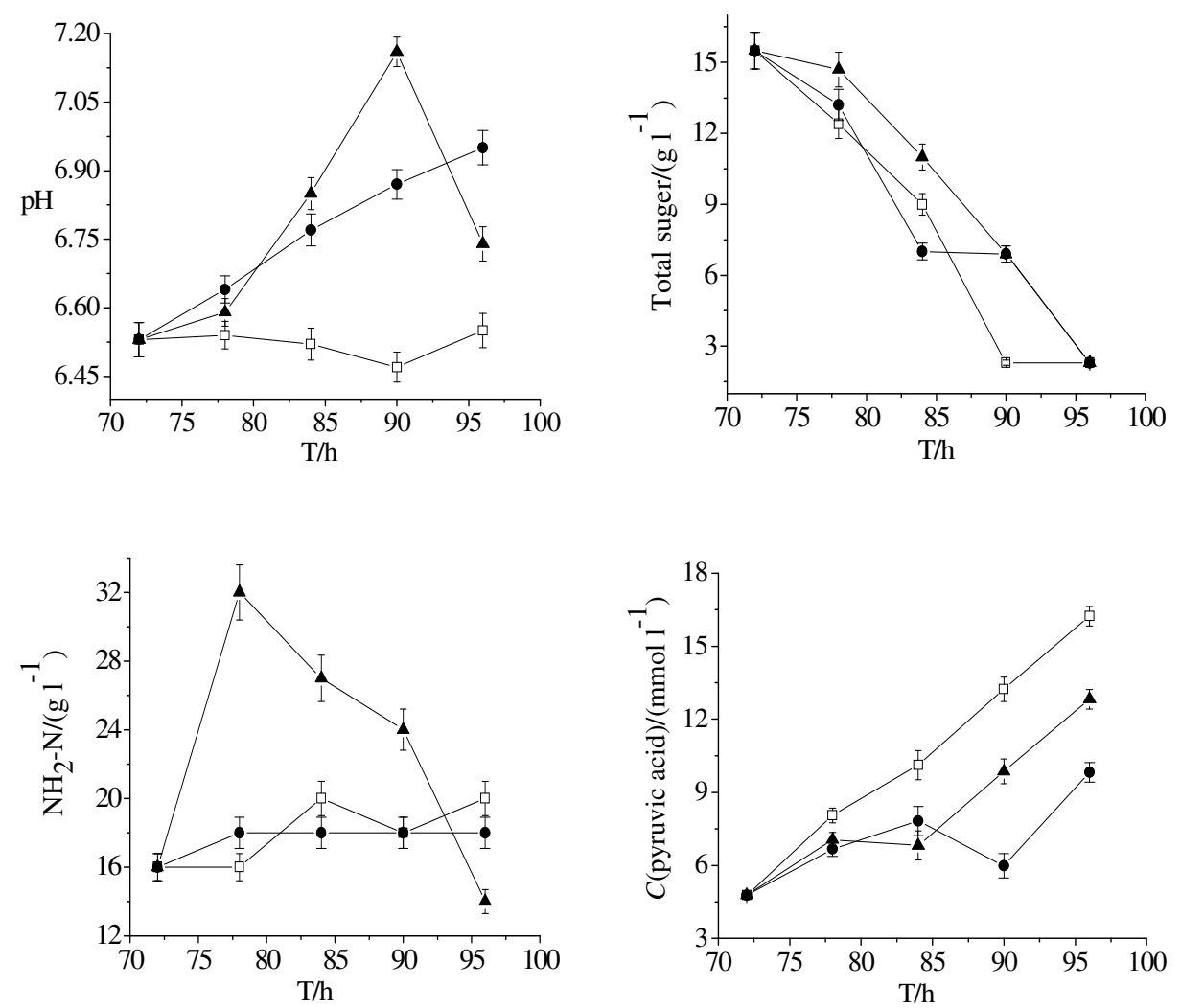

$\square \square-$ control $-\mathbf{\Delta}-$ Leu fed by single addition $-\bullet-$ Leu fed by multiple batches addition

Figure 7. Profiles of $\mathrm{pH}$, total sugar, $\mathrm{NH}_{2}-\mathrm{N}$ and intracellular pyruvate acid during bitespiramycin fermentation with different leucine feeding modes. Single addition: $2.0 \mathrm{~g} \mathrm{l}^{-1}$ Leu was supplemented once at $72 \mathrm{~h}$ of fermentation. Multiple batch addition: at $72 \mathrm{~h}$ of fermentation, a total of $2.0 \mathrm{~g} \mathrm{l}^{-1}$ Leu was fed at an interval of $6 \mathrm{~h}(72 \mathrm{~h}, 80 \mathrm{~h}$ and $90 \mathrm{~h}$ of fermentation).

\section{DISCUSSION}

Short-chain organic acids such as acetic acid, propionic acid and butyric acid, which are essential in the biosynthesis of lactone ring of macrolide antibiotics, are produced mainly by the EMP pathway, TCA cycle, and metabolism of fatty acids and amino acids. They play an important role in the primary and secondary metabolism of microorganism producing macrolide antibiotics, i.e., acting as a bridge between primary and secondary metabolisms. The results of this study indicate that the supplement of Val, Ile or Leu not only provided direct precursors for the biosynthesis of bitespiramycin, but also had various effects on the metabolism of microorganisms. 
The microorganism has different utilization patterns for each of the three amino acids, resulting in different effects on glycometabolism. It was observed that the $\mathrm{pH}$ of the culture medium did not increase after supplementation with Val, while it was higher than that of the control group after supplementation with Leu or Ile, and the concentration of ammonium ion in the fermentation broth had a marked increase after supplementation with Ile or Leu (Fig. 3A). During the metabolism process of Leu and Ile, the amino group was excreted in the culture broth after deamination reaction, and the remaining carbon skeleton was directly used by the bacteria cells. However, after supplementation with Val the concentration of ammonium ion in the fermentation broth became almost equal to that of the control group. A similar phenomenon was also observed by Voelker et al (23). They proposed that the amino group from the adsorbed Val was further used by mycerlia, and the remaining carbon skeleton, i.e., $\alpha$-ketoisovaleric acid, was temporarily excreted out and then re-adsorbed into the mycerlia for further use after the intracellular ammonium ions were exhausted.

It is reported that the transamination reaction of Val activates the enzymatic activity of glycometabolism (24), resulting in the increase of the glucose consumption rate. Meanwhile, the amino group transamination reaction of Val provides a $\mathrm{N}$ atom for the glycoside side chain of lactone ring of spiramycin (4). The change rule of the activity of PC and the content of intracellular pyruvic acid imply that supplementation of $\mathrm{Val}$ promotes the conversion of intracellular pyruvic acid into oxaloacetic acid, which is involved in the biosynthesis of malonyl-CoA and methylmalonyl-CoA. The above reaction not only provides direct precursors for the biosynthesis of lactone ring but also relieves the substrate inhibition effect, which converts redundant short-chain fatty acids such as acetyl-CoA and propionyl-CoA into malonyl-CoA and methylmalonyl-CoA via the catalysis of acyl carboxylases (4). Thus the biosynthesis of macro ring is promoted and the biological titer of bitespiramycin increases. In addition, after the supplementation of $\mathrm{Val}$, the 4"-O-butyryl group increased to some extent. Vezina et al (22) also observed that the biosynthesis of 4"-O-butyrylated components of kitasamycin (A4/A5) increased after Val feeding.

After supplementation of Ile, the glucose consumption rate and the biological titer of bitespiramycin decreased; however, the relative content of propionylspiramycin III, acetylspiramycin II and isobutyrylspiramycin increased significantly. Zhang et al (27) observed that because of the substrate inhibition effect of Ile on methylmalonyl-CoA racemase and allomerase, succinyl-CoA produced by TCA cycle was mainly excreted to the broth in the form of succinic acid, and could not be converted to the direct precursors of lactone ring. Ivan et al (9) demonstrated that the readsorption and reutilization of acetic acid and propionic acid were positively correlated to the activity of propionyl-CoA carboxylase. Therefore, although the carbon skeleton of Ile was adsorbed into the bacteria cells and then catabolized to produce abundant acetyl-CoA and propionyl-CoA, these acyl CoAs may not be converted to the direct precursors of lactone ring to produce bitespiramycin. In contrast, after supplementation with Ile, the relative content of propionylspiramycin III, acetylspiramycin II and isobutyrylspiramycin increased significantly, which means that short-chain organic acid-CoAs from Ile catabolism were transferred to the 4-OH of mycarose and the $3-\mathrm{OH}$ of macro ring (16).

The supplementation of Leu to the defined medium had no obvious effects on the biological titer of bitespiramycin, but resulted in the remarkable increase in the content of isovalerylspiramycins, indicating that isovaleryl-CoA derived from Leu can be used as the precursor of the $4 \square$ side chain of spiramycins. Vezina et al (22) also showed that the isovaleryl group of the A1/A3 component of kitasamycin came from leucine, and it was demonstrated that the isovaleryl side chain of carbomycin also came from Leu $(3,7)$. Leu catabolism can also provide an isovaleryl side chain for the biosynthesis of 3-O-acetyl-4"-O-isovaleryltylosin, when a tylosin-producing strain Streptomyces fradine was transformed with macrolide 4"-O-acyltransferase gene (2). The improvement effect of 
Leu on the biosynthesis of 4"- $O$-isovalerylspiramycins was further confirmed by supplementation with $2.0 \mathrm{~g} \mathrm{l}^{-1}$ Leu to the complex media, and the result shows that after batch supplementation of Leu the relative content of total isovalerylspiramycins increased from $31.4 \%$ to $46.9 \%$. This indicates that even with cultivation in complex media, the supply of isovaleryl-CoA is still a restriction factor significantly influencing isovaleryl spiramycin biosynthesis.

Although Val, Ile and Leu all belong to the group of branched-chain amino acids, they have different catabolism pathways within the microorganism, resulting in different effects on primary and secondary metabolism, and providing the possibility to regulate the composition and biological titer of bitespiramycin, a product of combinational biosynthesis, during the fermentation process.

\section{ACKNOWLEDGEMENTS}

The work was supported by the Scientific Research Foundation for the Returned Overseas Chinese Scholars and National Basic Research Program (973 Program No. 2007CB714303) and Shanghai Municipal Program for High Technology Research and Development (064319033). The authors also graciously acknowledge the Tonglian Group Shanghai Biotchenology Institute (China).

\section{REFERENCES}

1. Anon. (1995). Sterilization and sterility assurance of compendial articles. In U.S. Pharmacopeia 23rd edn. pp. 1976-1978. Rockville, MD: United States Pharmacopeial Convention, Inc.

2. Arisawa, A.; Kawamuna, N.; Naritn, T.; Kojima, I.; Okamura, K.; Tsunekawa, H.; Yochioka, T.; Okamoto, R. (1996). Direct fermentative production of acyltylosins by genetically-engineered stains of Streptomyces fradine. J Antibiot 49 (4): 349-354.

3. Arisawa, A.; Kawamuna, N.; Tsunekawa, H.; Okamura, K.; Tone, H.; Okamoto, R. (1993). Cloning and nucleotide sequence of two genes involved in the 4"-O-acylation of macrolide antibiotics from Streptomyces thermotolerans. Biosci Biotechnol Biochem 57 (12): 2020-2025.
4. Bai, X.F.; Tian, W.; Yu, X.L. (2003). Regulatory effects of ammonium ions on the biosynthesis of antibiotics. Chinese Medical Research Clinical 1 (1): 16-18.

5. Bartek, T.; Makus, P.; Klein, B.; Lang, S.; Oldiges, M. (2008). Influence of L-isoleucine and pantothenate auxotrophy for L-valine formation in Corynebacterium glutamicum revisited by metabolome analyses. Bioprocess Biosyst Eng. 31: 217-225.

6. Fortnagel, P.; Freese, E. (1969). Analysis of sporulation mutants. 2. Mutants blocked in the citric acid cycle. J Bacterial 95: 1434-1438

7. Grisebach, H.; Achenbach, H. (1963). On the origin of isovaleric acid in magnamycin. Experientia 19: 6-7.

8. Hafner, E.W.; Holley, B.W.; Holdom, K.S. (1991). Branched-chain fatty acid requirement for avermectin production by a mutant of Streptomyce avermitilis lacking branched-chain 2-oxo acid dehydrogenase activity. J Antibiot (Tokyo) 44 (3): 349-356.

9. Ivan, A.; Berg, L.; Filatova, V. (2002). Inhibition of acetate and propionate assimilation by itaconate via propionyl-CoA caboxylase in isocitrate lyase-negatibe purple bacterium Rhodospirillum rubrum. FEMS Microbiol. Lett. 216: 49-54.

10. Kirst, H.A.; Yeh, W.K.; Zmijewski Jr, M.J. (2001). Enzyme Technologies for Pharmaceutical and Biotechnological Applications. Marcel Dekker Inc pp: 89-111.

11. Krebs, H.A. (1970). The history of the tricarboxylic acid cycle. Perspect Biol Med 14: 154-170.

12. Lebrihi, A.; Germain, P.; Lefebvre, G. (1987). Phosphate repression of cephamycin and clavulanic acid production by Streptomyce clavuligerus. Appl Microbiol Biotechnol 26: 130-135.

13. Liu, L.M.; Li, Y. (2003). CaCO3 Stimulates $\alpha$-ketoglutarate accumulation during pyruvate fermentation by Torulopsis glabrata. Chin. J. Biotechnol. 19 (6): 745-749.

14. Lounes, A.; Lebrihi, A.; Benslimane, C. (1995). Regulation of L-valine catabolism by ammonium in Streptomyce ambifaciens producer of spiramycin. Can J Microbiol 41 (9): 800-807.

15. Miller, G.L. (1959). Use of dinitrosalicylic acid reagent for determination of reducing sugar. Anal Chem 31 (3): 426-430.

16. Miyagawa, K.; Suzuki, M.; Higaashide, E. (1979). Effect of aspartic acid family amino acid on production of maridomycin. Agric Biol Chem 43 (5): 1103-1109.

17. Pospisil, S.; Sedmera, P.; Krumphanzl, V. (1986). Biosynthesis of monensins A and B: the role of isoleucine. Folia Microbiol (Praha) 31 (1): 8-14.

18. Shang, G.D.; Dai, J.L.; Wang, Y.G. (2001). Construction and physiological studies on a stable bioengineered strain of shengjimycin. $J$ Antibiot (Tokyo) 54: 66-73.

19. Shi, X.G.; Fawcett, J.P.; Chen, X.Y.; Zhong, D.F. (2005). Structural identification of bitespiramycin metabolites in rat: A single oral dose study. Xenobiotica 35 (4): 343-358. 
Zhen-Lin, L. et al.

20. Shi, X.G.; Zhong, D.F.; Su, N.L. (2003). Pharmacokinetics of a novel antibiotic bitespiramycin in rats. Asian Journal of Drug Metabolism and Pharmacokinetics 3 (2): 134-137.

21. Shi, X.G.; Zhong, D.F.; Sun, Y.M.; Zhang, Y.F. (2004). Metabolites of a novel antibiotic bitespiramycin in rat urine and bile. Chinese Chemical Letters 15 (4): 431-434.

22. Vezina, C.; Bolduc, C.; Kudalski, A. (1979). Biosynthesis of kitasamycin (Leucomycin) by leucine analog resistant mutants of Streptomyces kitasatoensis. Antimicrob. Agents Chemother. 5 (5): 738746 .

23. Voelker, F.; Altaba, S. (2001). Nitrogen source governs the patterns of growth and pristinamycin production in Streptomyces pristinaespiralis. Microbiology 147, 2447-2459.

24. Wang, P.; Zhuang, Y.P.; Chu, J.; Zhang, S.L. (2005). Regulatory effects of ammonium ions on the biosynthesis of meilingmycin. Acta Microbiol. Sin. 45 (3): 405-409.
25. Weatherburn, M.V. (1976). Phenol-hypochloride reaction for determination of ammonium. Anal Chem 39: 971-974.

26. Yoshida, T.; Katagiri, K. (1967). Influence of isoleucine upon quinomycin biosynthesis by Streptomyces sp. 732. J. Bacteriol. 93 (4): 1327-1331.

27. Zhang, W.W.; Jiao, R.S. (1996). The regulation of methylmalonyl-CoA from formation pathways in rifamycin SV-producing Amycolatopsis Mediterranei U 32. Acta Microbiol. Sin. 36 (4): 276-283.

28. Zhang, X.H. (1991). The acylated spiramycin yield of spiramycin strain with acylase gene in Streptomyces ambofaciens 311-10. Chinese Journal of Antibiotics 16 (2): 315-322.

29. Zhong, D.F.; Shi, X.G.; Sun, L.; Chen, X.Y. (2003). Determination of three major components of bitespiramycin and their major active metabolites in rat plasma by liquid chromatography-ion trap mass spectrometry. Journal of Chromatography B - Analytical Technologies in the Biomedical and Life Science 791 (1-2): 45-53. 